\title{
Acoustic Emission Monitoring of Chemically Bonded Anchors
}

\author{
Piervincenzo Rizzo • Antonino Spada • Sandeep Degala • \\ Giuseppe Giambanco
}

Published online: 12 February 2010

(C) Springer Science+Business Media, LLC 2010

\begin{abstract}
This paper presents a study on the use of acoustic emission (AE) to assess the structural soundness of concrete reinforced with chemically bonded anchors. The results of an experimental work based on six pullout tests monitored using an $\mathrm{AE}$ instrumentation suite are reported below. In every test one rebar was embedded in the hardened concrete by means of polyester resin. The AE was adopted to monitor the onset and progression of any structural damage. The parametric analysis, the intensity analysis and the moment tensor analysis of AE data were used to discriminate among different sources of damage. The technique shows promise for field application and may contribute to fully understand the structural mechanism in the rebar/adhesive/concrete systems.
\end{abstract}

Keywords Anchor rods $\cdot$ Pullout tests $\cdot$ Acoustic emission $\cdot$ Parameter analysis $\cdot$ Moment tensor $\cdot$ Intensity analysis

P. Rizzo $(\bowtie) \cdot$ A. Spada $\cdot$ S. Degala

Laboratory for NDE and SHM studies, Department of Civil and Environmental Engineering, University of Pittsburgh, 942 Benedum Hall, 3700 O'Hara Street, Pittsburgh, PA 15261, USA

e-mail: pir3@pitt.edu

A. Spada · G. Giambanco

Department of Structural and Geotechnical Engineering,

University of Palermo, Viale delle Scienze, Palermo, 90128, Italy

Present address:

S. Degala

DIETRICH Design Group, A Worthington Industries Company,

1414 Field St., Building C, Suite 1, Hammond, IN 46320, USA

\section{Introduction}

Epoxy adhesive anchor systems are widely used in civil engineering to anchor both threaded rods and reinforcing bars into hardened concrete. Common applications include bridge widening, structure-mounted signs, luminaries and light poles, concrete repair and rehabilitation, and tunneling finishing. The recent collapse of a section of a suspended concrete ceiling of the Interstate 90 connector tunnel in Boston due to a poor creep resistance of the epoxy resin and a general lack of understanding the effects of creep in epoxy adhesive anchor systems [1] has demonstrated the importance of develop an in-situ nondestructive method to monitor such structural systems.

Anchors can be divided into two general groups: castin-place and post-installed. A typical cast-in-place anchor consists of a rebar in concrete with or without protective coating. Instead a post-installed anchor is made of a rebar installed in a hole and then bonded with a chemical or a nonchemical agent. Bonded anchors utilizing nonchemical agents are often called "grouted anchors". To evaluate the structural strength of anchor systems the destructive method of "pullout test" is commonly used. Using this test, the force needed to pull out an anchor is estimated.

To perform in-situ evaluation of epoxy bonded anchors, nondestructive evaluation (NDE) methods are necessary. To the best of the authors' knowledge, no literature is available about NDE of epoxy bonded anchor systems. Typically, previous works focused on rebar/concrete systems. Cheng and Chiou [2] and Lin et al. [3] used pullout tests and impactecho method to estimate the bond-loss at the interface between steel bars and concrete. Ghandehari and co-authors [4] used speckle laser interferometry to monitor the displacements at the concrete/rebar interface and to measure crack length and crack opening during a pullout test. Video 
microscopy was used by Söylev and François [5] during the pullout test of bars from concrete column elements. X-rays are used to visualize the failure in a pullout test of cable bolts and rock bolts [6]. Raman spectroscopy was applied by Pisanova et al. [7] to monitor the crack length during the pullout of glass fibers or aramid fibers from epoxy. The active method of guided ultrasonic waves was numerically and experimentally investigated by Beard et al. [8] and Beard and Lowe [9] to detect the presence of damage in rebars embedded in concrete. The passive method of acoustic emission (AE) was applied to pullout tests in the bar/concrete systems [10-13]. For instance, Balázs et al. [10] discussed AE results obtained in monotonic loading, cyclic loading and long-term loading of rebars embedded in concrete cubes and also subjected to a pullout load.

In this paper, the results of experimental pullout tests conducted on post-installed, chemically bonded anchors are presented. Each anchor consisted of a rebar chemically bonded to a hardened concrete cylinder by means of polyester resin. The specimens were monitored using an $\mathrm{AE}$ suite instrumentation. In the light of the previous researches, the main objective of the present paper is to demonstrate the use of $\mathrm{AE}$ for failure monitoring of adhesively bonded anchor rods. By attributing each emission to a particular source type or failure mode, the resulting different sources of damage can be discriminated.

Six pullout tests were performed. The AE data were analyzed using the parameter analysis [14-16], the intensity analysis [16, 17], and the moment tensor analysis [18-22].

It is demonstrated that by combining a few different $\mathrm{AE}$ approaches, it is possible to discriminate between different sources of damage in adhesively bonded anchor systems.

\section{The Pullout Test: Generalities and Failure Modes}

The pullout test measures the force required to pull an embedded bar from a concrete specimen or a given structure [23].

While in the cast-in-place anchors the load is transferred into the concrete at the anchor head, in adhesive anchors, the load is transferred from the steel through the adhesive layer along the bonded surface. The bond at the interface consists of three mechanisms: adhesion, friction, and mechanical interlocks.

In pristine conditions, the bond between steel and the adhesive medium or concrete is due to the chemical adhesion. Once the chemical connections fail and relative displacements occur between the two surfaces the friction forces would dominate. If debonding takes place around the rebar surface, the ribs govern the stress-deformation behavior. When the resin between the ribs is subjected to a shear force, its deformation creates a bar-resin mechanical interlock dependent on the ribs' geometry. The inclined shape of

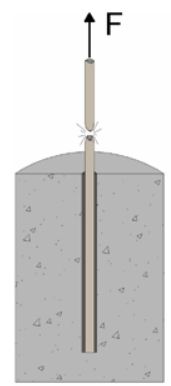

(a)

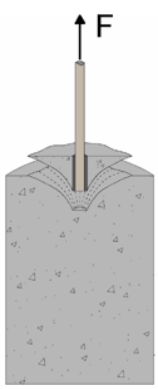

(b)

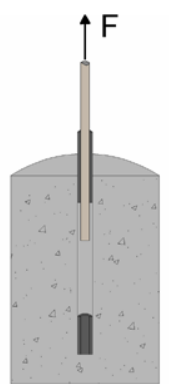

(c)

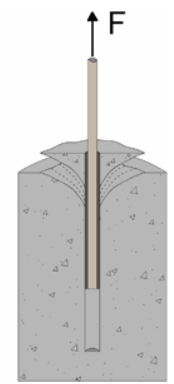

(d)
Fig. 1 Failure modes during a pullout test for unconfined concrete: (a) steel failure; (b) concrete cone failure; (c) bond failure; (d) combined cone-bond failure

the ribs is therefore subjected to a force orthogonal to the ribs' surface which turns into a reaction acting on the resin or concrete.

When the rebar is cast-in-place without coating, at the early stages of a pullout test, inclined cracks (theoretically at $45^{\circ}$ ) start to appear due to pure shear stress in the concrete which yields to tensile and compressive stresses along the principal directions. When the load increases, the horizontal component of the reaction force represents an increasing radial force that, for large slip values, promotes the development of longitudinal cracks. This mode of failure is usually referred to as a splitting failure.

Rebars chemically bonded offer higher limit stress with respect to the cast-in-place systems. Such systems are prone to one of the following failure modes [24, 25]:

1) an anchor steel failure characterized by yielding or fracture of the steel;

2) a concrete cone failure occurring when the embedment's depth is less than $50 \mathrm{~mm}$ and the concrete is unconfined;

3) a bond failure at the bar/resin or resin/concrete interface;

4) a combined cone-bond failure usually in unconfined concrete and an embedment depth greater than 50-100 mm.

These failures are schematized in Fig. 1. The occurrence is dependent upon boundary and anchoring conditions, the bonding agent and the materials involved, the surface roughness, the temperature, and the loading gradient [24, 25].

\section{Acoustic Emission}

AE method exploits the propagation of transient elastic waves generated by the rapid release of energy from a localized source or sources within a specific material. The elastic energy propagates as a stress wave (AE event) in the structure and is detected by one or more $\mathrm{AE}$ sensors. $\mathrm{AE}$ events may be generated by moving dislocations, crack onset growth and propagation, fiber breaks, disbonds, plastic deformations, etc. 
The conventional parameter analysis of an AE event evaluates $\mathrm{AE}$ features such as counts, amplitude, rise time, energy, etc. These parameters are then correlated to the mechanical/structural event that generated the transient wave.

Closely related to the parameter analysis, the intensity analysis (IA) evaluates the structural significance of an $\mathrm{AE}$ event as well as the level of deterioration of a structure by calculating two values called the historic index $(H I)$ and severity $\left(S_{r}\right)[16,17]$. The intensity is then found by plotting the maximum of each value on a chart. The HI compares the signal strength of the most recent emissions to the signal strength of all emissions. Analytically, the $\mathrm{HI}$ and the $S_{r}$ are defined as:

$H I=\frac{N}{N-K}\left(\frac{\sum_{i=K+1}^{N} S_{o i}}{\sum_{i=1}^{N} S_{o i}}\right) S_{r}=\frac{1}{J} \sum_{m=1}^{J} S_{o m}$

where $N$ is number of AE emissions (referred to as "hits") up to time $t ; S_{o i}$ is the signal strength of the $i$ th event; $K$ and $J$ are empirical constants based on the material under investigation. In the present paper, the following values for $K$ and $J$ are used: for $N \leq 100, K$ is not applicable; for $101<$ $N<500, K=0.8 N$; and for $N>500, K=N-100$; for $N \leq 20 J$ is not applicable whereas for $N>20, J=20$ $[16,17]$.

The presence of one or more peaks may reveal the occurrence of new damage or the propagation of damages, respectively. The severity is the average of the $J$ largest signal strength emissions received at a sensor. As the severity is a measure of structural damage, an increase in severity often corresponds to new structural damage.

\subsection{Source Localization}

The location of AE sources can be determined by comparing the differences among the time of arrival of the elastic waves at the $\mathrm{AE}$ sensors. In bulk geometries the propagation of longitudinal waves (p-waves) is exploited. Let us assume that a bulk isotropic structure is monitored by using $n \mathrm{AE}$ transducers. The time of arrival $t_{s, i}$ of the p-wave at sensor (s) can be written as:

$$
\begin{aligned}
t_{s, i} & =\frac{\left|\mathbf{r}_{s, i}-\mathbf{r}\right|}{v_{p}}+T \\
& =\frac{\sqrt{\left(x_{s, i}-x\right)^{2}+\left(y_{s, i}-y\right)^{2}+\left(z_{s, i}-z\right)^{2}}}{v_{p}}+T
\end{aligned}
$$

where $\mathbf{r}_{s, i}=\left(x_{s, i}, y_{s, i}, z_{s, i}\right)$ is the position vectors of sensor $(i=1, \ldots, n), \mathbf{r}=(x, y, z)$ is the position vector of the $\mathrm{AE}$ source, and $v_{p}$ is the velocity of the longitudinal bulk wave. Equation (2) assumes that the wave velocity is independent on the direction of propagation and that the wave is not dispersive. $T$ identifies the instant at which the release of transient energy occurred.

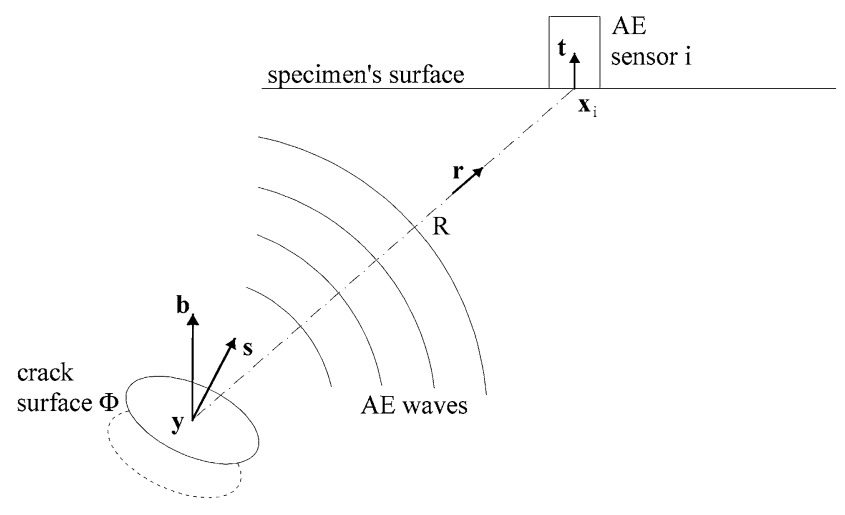

Fig. 2 Formation, propagation and acquisition of an AE event

In (2) the location of the sensors is known and the arrival time $t_{s, i}$ is measured. As (2) contains four unknowns, the source coordinates and the instant $T$, at least four sensors are necessary. When more than four sensors are installed, the problem is over-determined but the result is more accurate. Moreover the problem is non-linear. In the present work the Levenberg-Marquardt method was used to solve the unknowns. The method is a non-linear least squares approach [26] that uses an iteration algorithm where the estimation of the solution is computed by minimizing the errors of the unknown parameters.

\subsection{Moment Tensor Analysis}

The Simplified Green's function for Moment tensor Analysis (SiGMA) proposed by Ohtsu [19] was applied to determine the orientation, direction and volume of cracks generated during the pullout tests.

Let consider a crack motion vector $\mathbf{b}(\mathbf{y}, t)$, at a point $\mathbf{y}$ and instant $t$, of a fracture surface $\Phi$ with normal vector $\mathbf{s}$ as depicted in Fig. 2. When $\mathbf{b}$ is parallel to $\mathbf{s}$ a tensile crack is propagating. Conversely, when $\mathbf{b}$ is orthogonal to $\mathbf{s}$ a shear crack is generated. Because vector $\mathbf{b}$ is time-dependent, its variation with time generates the elastic wave $\mathbf{u}_{i}\left(\mathbf{x}_{i}, t\right)$ detected by the AE transducers [19]. If $\mathbf{b}(\mathbf{y}, t)$ is decomposed as:

$\mathbf{b}(\mathbf{y}, t)=b(\mathbf{y}) \mathbf{l} S(t)$

with $\mathbf{I}$ the unit vector of the crack motion, $b(\mathbf{y})$ the magnitude of the crack displacement at point $\mathbf{y}$ and $S(t)$ the source time function of crack motion, the theoretical waveform at sensor $i$ can be written as:

$\mathbf{u}_{i}\left(\mathbf{x}_{i}, t\right)=\int_{\Phi} G_{i p, q}\left(\mathbf{x}_{i}, \mathbf{y}, t\right) C_{p q k l} b(\mathbf{y}) l_{k} n_{l} * S(t) d \Phi$

where $G_{i p, q}$ is the spatial derivative of Green's function, $C_{p q k l}$ are the elastic constants of the material, and the symbol * represents the convolution integral. Assuming:

$m_{p q}=C_{p q k l} l_{k} n_{l} \Delta V$ 
where

$\Delta V=\int_{\Phi} b(\mathbf{y}) d \Phi$

is the crack volume, (4) can be rewritten as:

$\mathbf{u}_{i}\left(\mathbf{x}_{i}, t\right)=G_{i p, q}\left(\mathbf{x}_{i}, \mathbf{y}, t\right) m_{p q} * S(t)$

where $m_{p q}$ is called moment tensor. The elements $m_{p q}$ of the tensor are the product of a volume by a stress and therefore, they have the unit of a moment.

In the SiGMA approach, only the amplitude of the first cycle of the AE waveform is considered. Thus, (7) is simplified as [21]:

$$
\begin{aligned}
A(\mathbf{x})= & \frac{C_{s} \operatorname{Ref}(\mathbf{t}, \mathbf{r})}{R}\left(\begin{array}{lll}
r_{1} & r_{2} & r_{3}
\end{array}\right)\left(\begin{array}{lll}
m_{11} & m_{12} & m_{13} \\
m_{21} & m_{22} & m_{23} \\
m_{31} & m_{32} & m_{33}
\end{array}\right) \\
& \times\left(\begin{array}{l}
r_{1} \\
r_{2} \\
r_{3}
\end{array}\right)
\end{aligned}
$$

where $A(\mathbf{x})$ is the amplitude of the first motion, $C_{S}$ is the calibration coefficient of the sensor, and $\operatorname{Ref}(\mathbf{t}, \mathbf{r})$ is the reflection coefficient between vector $\mathbf{t}$ and vector $\mathbf{r}$. As shown in Fig. 2, the vector $\mathbf{t}$ is the direction of AE transducer sensitivity, whereas the vector $\mathbf{r}$ represents the unit vector that identifies the wave propagation direction from the source to the sensor, separated by the distance $R$.

In (8), vector $\mathbf{t}$ and the waveform amplitudes recorded at each sensor are known. If the AE transducers used for the localization are identical, i.e., they possess the same sensitivity, then the coefficient $C_{S}$ can be neglected. The moment tensor $m_{p q}$ is symmetric and only six elements are independent. The distance $R$ and the vector $\mathbf{r}$ are known after source localization. Therefore, (8) has six unknowns and at least six sensors are needed to calculate the elements $m_{p q}$.

Once the elements $m_{p q}$ are determined the eigenvalue analysis of the moment tensor is performed. This analysis yields to the determination of the crack type, orientation, and direction. From the tensor eigenvalues $\lambda_{1}, \lambda_{2}$ and $\lambda_{3}$, with $\lambda_{1}>\lambda_{2}>\lambda_{3}$, the values of the shear ratio $X$, deviatoric tensile ratio $Y$, and isotropic tensile ratio $Z$ can be calculated by solving the following system

$\left\{\begin{array}{l}\frac{\lambda_{1}}{\lambda_{1}}=1=X+Y+Z \\ \frac{\lambda_{2}}{\lambda_{1}}=0-0.5 Y+Z \\ \frac{\lambda_{3}}{\lambda_{1}}=-X-0.5 Y+Z\end{array}\right.$

When $X>60 \%$ the crack is referred to as a shear crack; when $X<40 \%$ and contemporary $Y+Z>60 \%$ the sources are referred to as tensile cracks; finally, if $40 \%<X<60 \%$ the source is considered as a mixed crack $[19,21]$. (a)

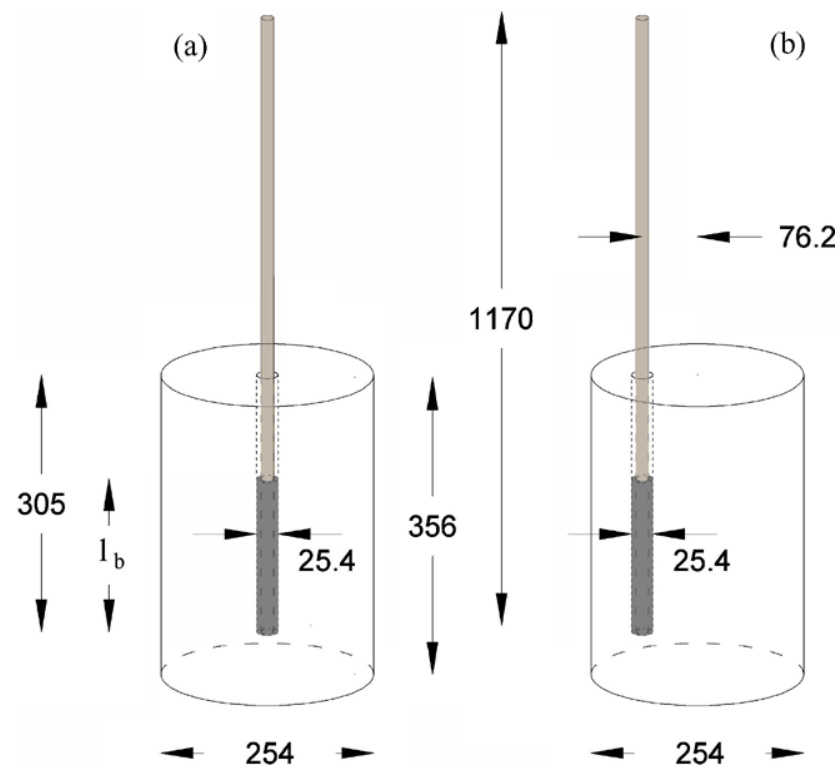

Fig. 3 Geometric characteristics of the tested specimens. (a) Rebar centrally embedded in the hardened concrete. (b) Rebar eccentrically embedded in the hardened concrete. Dimensions in $\mathrm{mm}$

The computation of the eigenvectors $\mathbf{e}_{1}, \mathbf{e}_{2}$ and $\mathbf{e}_{3}$ yields to the determination of the unit crack motion vector and the unit crack normal vector by means of the following system:

$\left\{\begin{array}{l}\mathbf{e}_{1}=\mathbf{l}+\mathbf{s} \\ \mathbf{e}_{2}=\mathbf{l} \times \mathbf{s} \\ \mathbf{e}_{3}=\mathbf{l}-\mathbf{s}\end{array}\right.$

where $\times$ is the vector product.

\section{Experimental Setup}

Six concrete cylinders were cast using water/cement ratio equal to 0.4 . The cylinders were $254 \mathrm{~mm}$ in diameter and $356 \mathrm{~mm}$ high (Fig. 3).

Two rebar sizes were considered, namely rebar \#5 $\left(197.93 \mathrm{~mm}^{2}\right)$ and \#6 (285.02 $\left.\mathrm{mm}^{2}\right)$ section. Each rebar was $1170 \mathrm{~mm}$ long. The nominal yielding and ultimate forces for rebar \#5 were of $84.4 \mathrm{kN}$ and $126.6 \mathrm{kN}$, respectively, and $117.5 \mathrm{kN}$ and $176.2 \mathrm{kN}$, respectively, for rebar \#6.

After curing, each cylinder was drilled to create a $25.4 \mathrm{~mm}$ diameter hole, $305 \mathrm{~mm}$ deep. Every hole was flushed with water to remove the dust. Once the holes were dry, a rebar was anchored in the concrete by using Minova Lokset Polyester Resin. The bond length $l_{b}$ and the geometric characteristics of the six specimens are schematized in Fig. 3 and summarized in Table 1.

The pullout test was performed after 24-27 hours of resin curing time using a universal Baldwin servo-hydraulic machine operated in displacement control. A schematic view of the entire test arrangement is shown in Fig. 4. 
Table 1 Geometric characteristics and main pullout tests' data

\begin{tabular}{lllllll}
\hline Test No & $l_{b}(\mathrm{~mm})$ & $\begin{array}{l}\text { Rebar size/centric }(\mathrm{C}) \\
\text { or eccentric }(\mathrm{E})\end{array}$ & Disp rate time $(\mathrm{mm} / \mathrm{s})$ & Peak load $(\mathrm{kN})$ & Failure type & Notes \\
\hline 1 & 152 & $\# 5 / \mathrm{C}$ & 0.091 & 71.30 & debonding & $/$ \\
2 & 152 & $\# 5 / \mathrm{C}$ & 0.120 & 110.80 & debonding & Rebar and cylinder are the same \\
& & & & & co Test 1 \\
3 & 305 & $\# 5 / \mathrm{E}$ & 0.082 & 87.89 & concrete splitting $/$ & $/$ \\
4 & 184 & $\# 6 / \mathrm{C}$ & 0.013 & 146.55 & debonding & / \\
5 & 152 & $\# 5 / \mathrm{C}$ & 0.053 & 84.52 & debonding & Rebar is the same of Test 5 \\
\hline
\end{tabular}

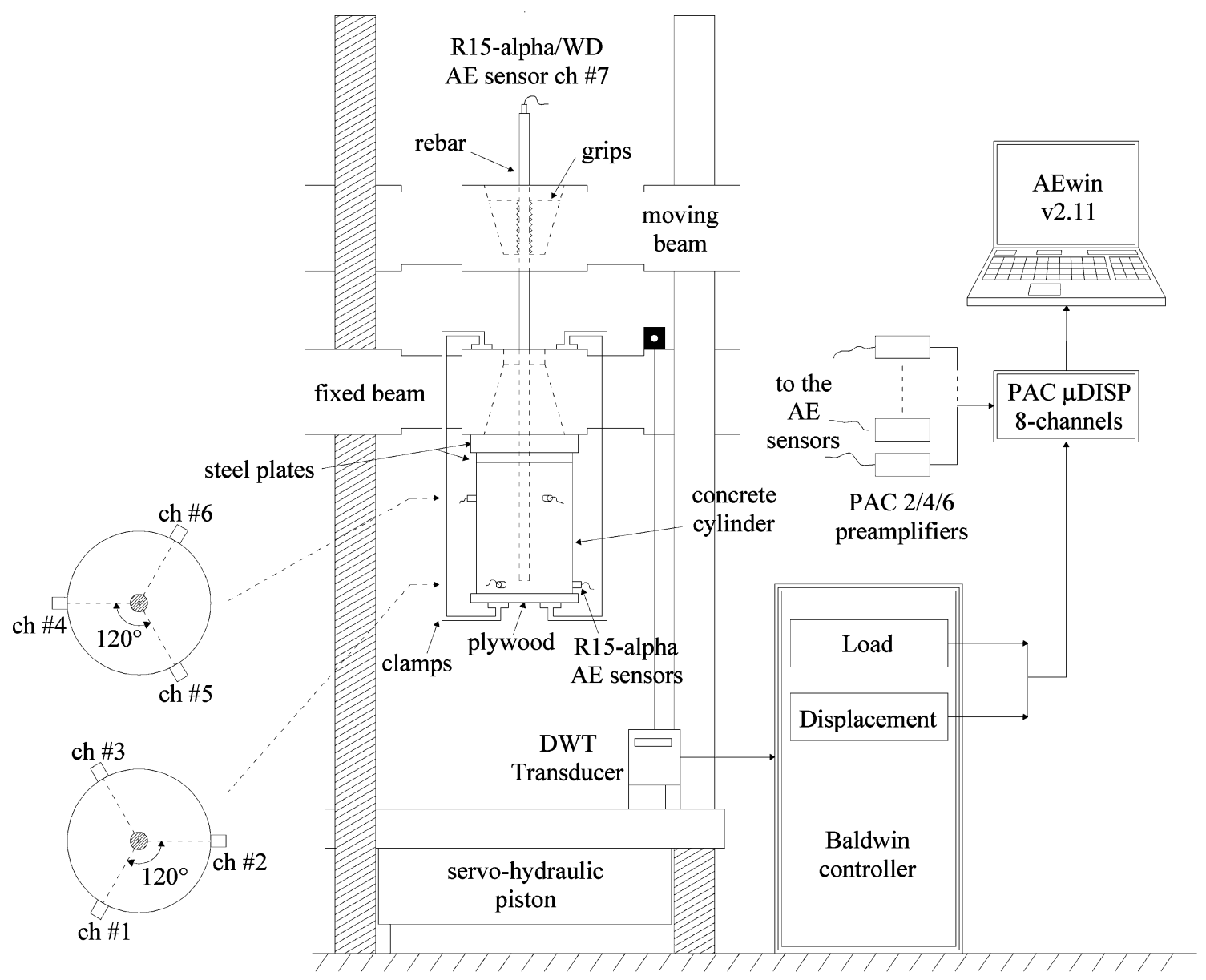

Fig. 4 Schematic view of the test setup

The cylinders were clamped to the fixed lower crossbeam of the machine. Two squared steel plates were positioned between the cylinder and the crossbeam in order to center the bar and provide a uniformly distributed stress on the top surface of the concrete. The relative displacement between the two crossbeams was measured by a DWT transducer with $0.1 \mathrm{~mm}$ resolution. The applied displacement rate time, the peak load, and the observed failure are given in Table 1.
The AE instrumentation consisted of: 1) broadband $\mathrm{AE}$ piezoelectric transducers (Physical Acoustics R15-alpha and WD transducers) used in conjunction with preamplifiers set at a $40 \mathrm{~dB}$ gain; 2) an eight-channel high-speed Physical Acoustics $\mu$ DiSP data acquisition board; 3) laptop with dedicated AEwin v2.11 software for signal processing and storage.

Prior to the tests, transducer positioning, signal threshold settings, and sensor sensitivity were determined using the 


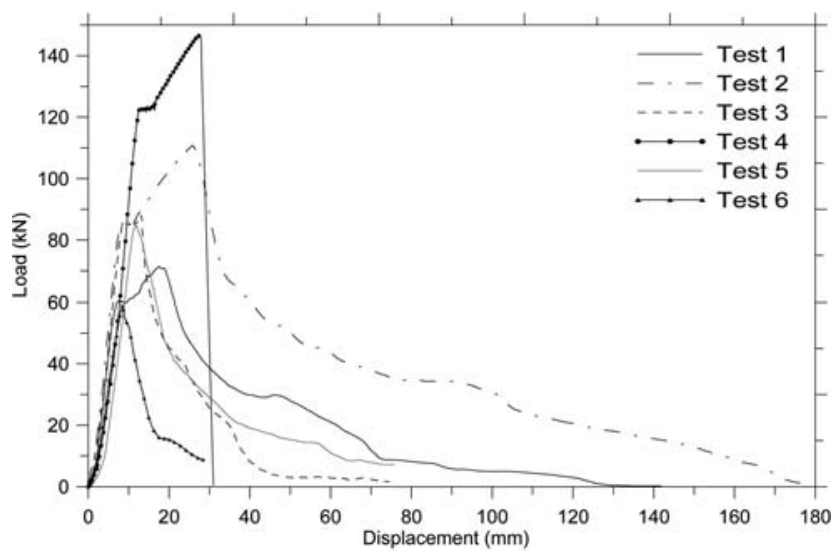

Fig. 5 Pullout test results: load-displacement curves

pencil lead break test. The threshold level was set at $40 \mathrm{~dB}$. The sampling rate was $5 \mathrm{MHz}$. One WD sensor was attached on the cross-section end of the rebar for Tests $1-5$, while an R-15 was used in Test 6 . Six R15 transducers were glued on the concrete cylinders to monitor Tests 5-6.

\section{Experimental Results: Parametric and Intensity Analysis}

During a pullout test, AE can be generated in the rebar, due to its deformation, at the rebar/resin or resin/concrete interfaces due to debonding, in the concrete or in the resin, due to micro or macro-cracking, in correspondence with the grips due to the frictional effects, or at the steel plate/concrete contact surface due to concrete crushing.

The load-displacement curves obtained for all the tests are shown in Fig. 5. A small nonlinear curve is visible at the beginning of every test, due to the start-up of the machine and to the grips' adjustment.

\subsection{Test 1}

Test 1 was characterized by an elastic regime up to about $60 \mathrm{kN}$, followed by a partial loss of bond until the peak load of $71.30 \mathrm{kN}$ was reached (Fig. 5). At the peak micro-cracks started to coalesce in macro-cracks and a softening branch took place until a complete debonding at the rebar/resin interface was obtained. Therefore, the system failed due to shear pullout.

Counts, amplitude, and duration are plotted as functions of time in Fig. 6. The plot of load versus time is superimposed in Fig. 6a. As for Tests 2, 3 and 4, only one sensor on the top of the rebar was used. The activity observed during the elastic regime is mostly generated by the elastic deformation on the rebar and at the grips, and in part by micro-cracking at the rebar/resin interface and crushing and
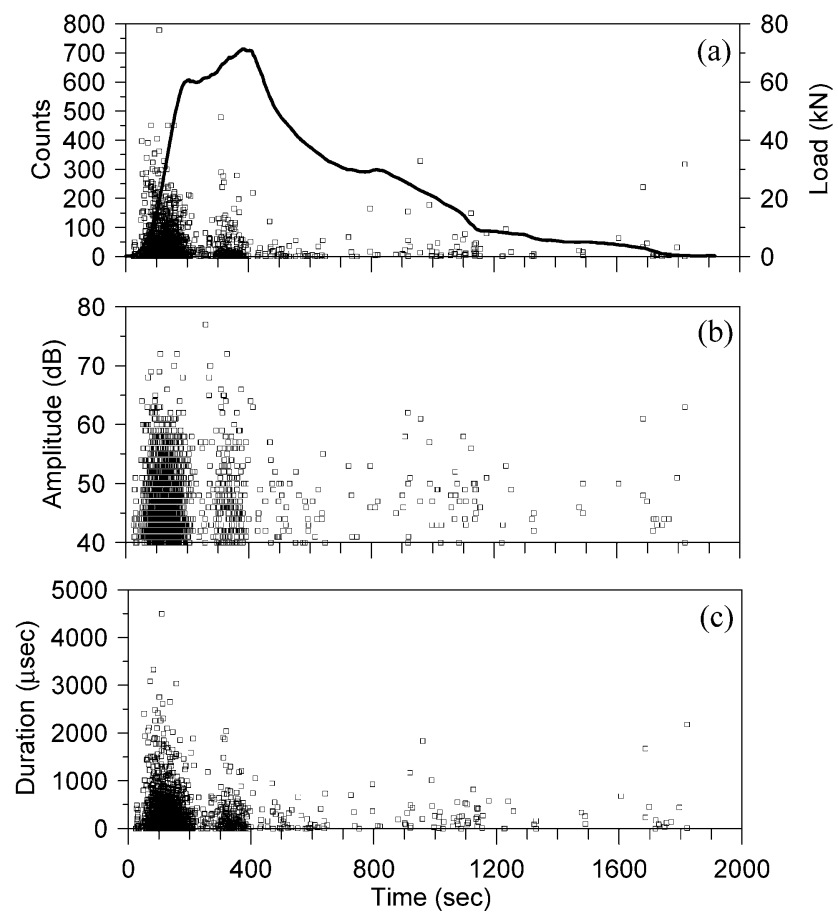

Fig. 6 Test 1-parametric analysis results: (a) counts and applied load, (b) amplitude and (c) duration as a function of time

friction associated with the contact forces between the concrete and the steel plate. Lesser activities were visible between 200 and 300 seconds. As in this range, the load was slightly lower, and the Kaiser effect took place. The Kaiser effect is the irreversible phenomenon of $\mathrm{AE}$ according to which, if a load is released on a structure and then gradually reapplied, no additional $\mathrm{AE}$ will be generated until the load exceeds the previous maximum value. Therefore, the phase comprised between 200 and 300 seconds was characterized by transient energy released at the bond interface. It can be noted that these emissions presented a lower duration and rise time (not shown), typical of an impulsive source. When the load exceeded the previous peak, new AE were detected until the debonding took place. After the debonding, because of the Kaiser effect the acoustic activity was mainly generated at the rebar/resin interface and related to a combination of debonding and frictional effects. Overall, less frequent events can be correlated with activities at the bond interface; more frequent events with longer duration can be associated with activities on the rebar. The longer $\mathrm{AE}$ duration is, the longer the consequence of the multiple reflections and the low energy attenuation occurring in the rebar would be.

Figure 7 shows the HI, the cumulative signal strength (CSS) and the severity as functions of time. The load curve is also superimposed on the severity plot. Both the CSS and the severity show patterns very similar to the load curve. Two knees can be noted at around 200 seconds and $400 \mathrm{sec}-$ onds respectively, when the debonding takes place. More- 

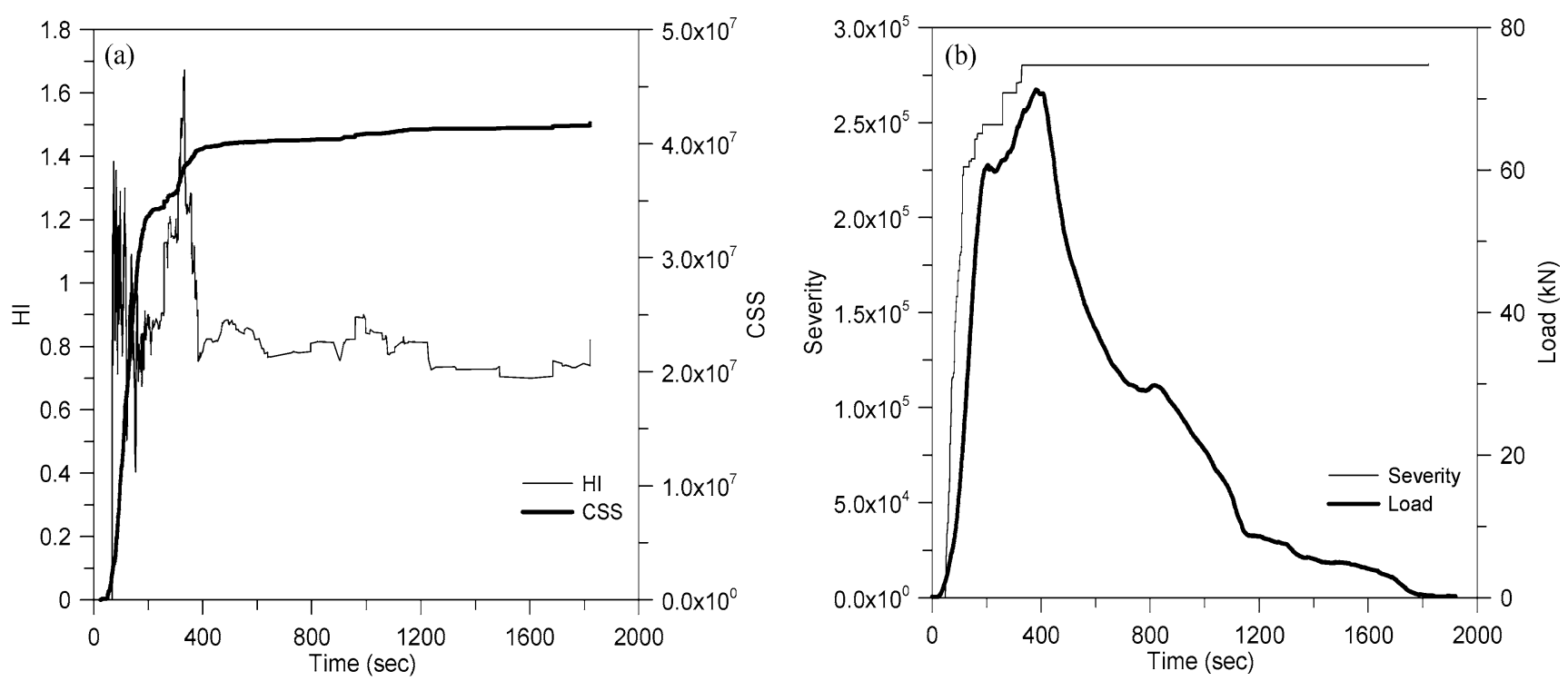

Fig. 7 Test 1—intensity analysis results: (a) HI and CSS plots; (b) severity

over, the $\mathrm{HI}$ index clearly shows the changings in the structure in the elastic range and between 200 and 400 seconds. The HI continues to change even during the debonding, but with fewer fluctuations. It can be argued that higher fluctuations are correlated to the emissions in the rebar, while shorter variations are attributed to the emissions at the bond. Thus, the HI can be considered as an analytical method of estimating the changes of slope in the CSS against time by comparing the signal strength of the most recent hits to all the hits for a given AE channel.

\subsection{Test 2}

In order to exploit the Kaiser effect, avoid the events associated with the contact forces between the plate and the concrete top surface, and isolate the sources at the rebar/resin interface up to the load of $71.30 \mathrm{kN}$, the same rebar and cylinder used in Test 1 were reused for Test 2. From Fig. 8 it is visible that the rebar reached its yielding load at about $85 \mathrm{kN}$. Yielding was followed by hardening until $110 \mathrm{kN}$, until the debonding initiated the softening branch. The system failed for shear pullout failure at the rebar/resin interface.

Counts, amplitude, and duration are plotted as a function of time in Fig. 8. The plot of total applied load is also superimposed on the plot of acoustic count history. As expected, fewer AE events with respect to Test 1 were detected during the initial elastic range. These events were generated at the grips and by transient energy released mainly by microcracking at the bond interface.

Once the previous peak load of $71.30 \mathrm{kN}$ was exceeded, large amount of activities were sensed until the hardening phase began because of the new events occurring in the rebar. Higher values of counts, amplitudes, duration, and rise
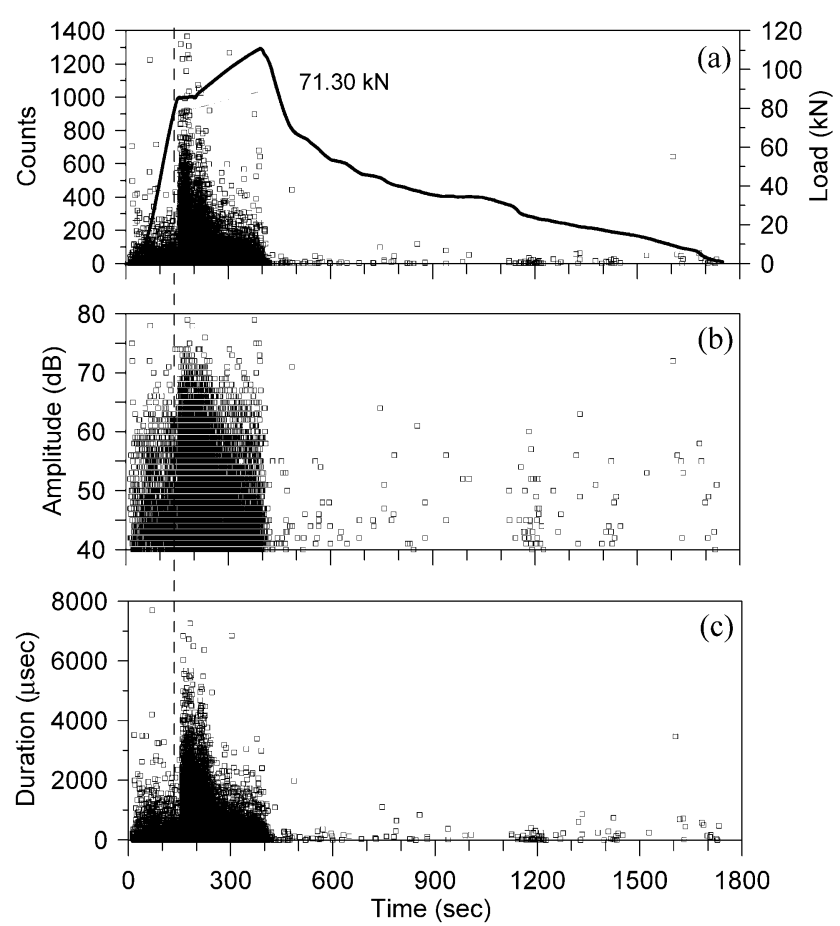

Fig. 8 Test 2-parametric analysis results: (a) counts and applied load, (b) amplitude and (c) duration as a function of time

time (not shown) are visible in Fig. 8. The frequency of the events instead dramatically decreased during the softening branch, when macro-cracks are forming and no more activity from the rebar can be generated due to the Kaiser effect.

The results of the intensity analysis are shown in Fig. 9. The CSS clearly shows a sudden change of the slope at about 200 seconds, which is due to the occurrence of a damaged mechanism. The HI curve shows a dense area of high value spikes. Such spikes are related to rebar yieldings. In 

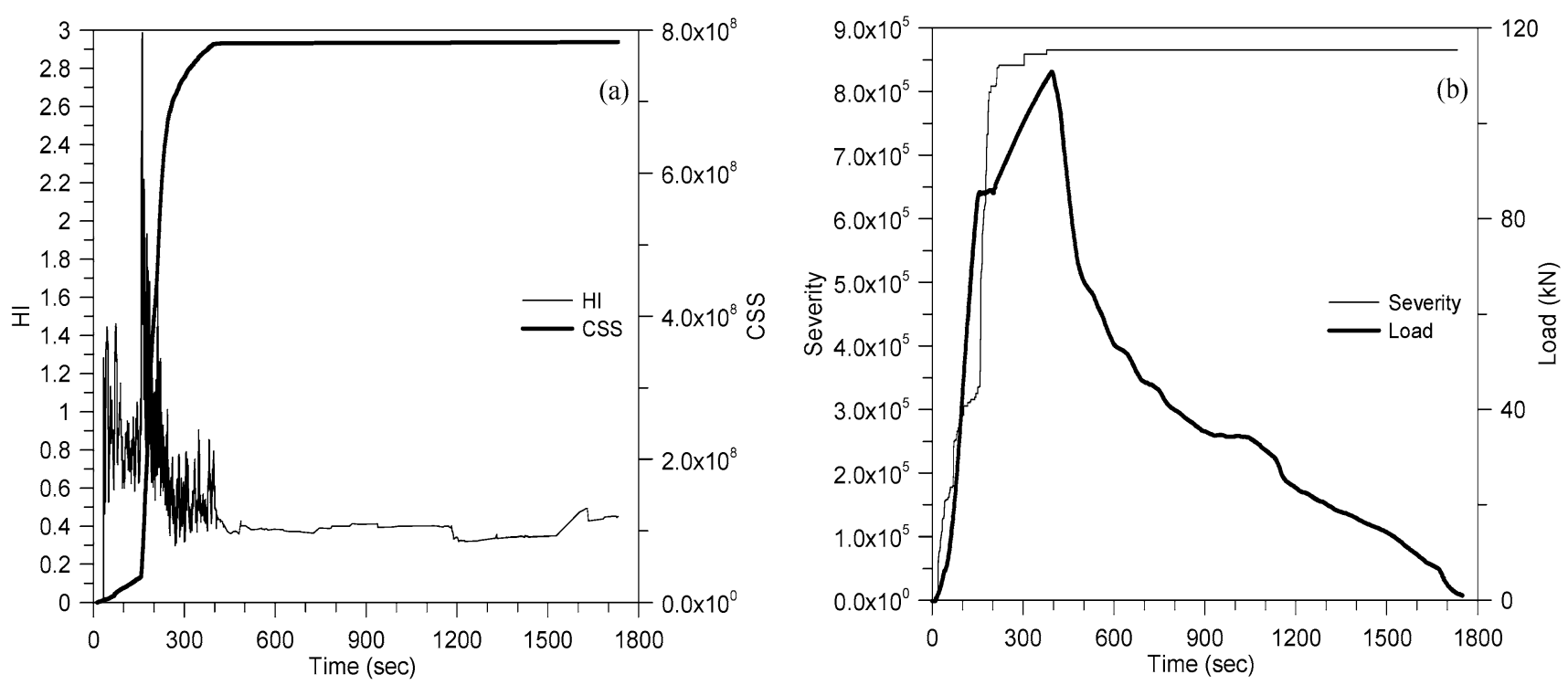

Fig. 9 Test 2-intensity analysis results: (a) HI and CSS plots; (b) severity

the range interval between 230 and 400 seconds the spikes are associated with rebar hardening and formation of new micro-cracks. Finally, above 400 seconds the constant value of CSS, severity, and HI denotes that structural failure had already occurred.

\subsection{Test 3}

In Test 3, the rebar was fully bonded into the concrete, $76.2 \mathrm{~mm}$ away from the center of the cylinder. The loaddisplacement curve is reported in Fig. 5. The development of longitudinal cracks in the concrete (splitting) due to the stress concentration in the outer portion of concrete occurred during the rebar's hardening phase.

Figure 10 shows counts, cumulative energy, duration, HI and CSS reported as functions of time. The applied load is superimposed on the counts' plot. The first portion of the test is dominated by grips' adjustment and friction between the grips and the rebar. Overall, five different zones can be identified. In the first zone, the elastic behavior of the structure is accompanied by events associated with the rebar elongation and development of micro-cracks at the bond interface. This is confirmed by the rapid fluctuations of the HI (Fig. 10d). At the yielding phase, the plastic deformation of the rebar induces the generation of more events. The slope change in the plot of the cumulative energy and the CSS is visible. The HI shows more frequent spikes as well. The third zone is characterized by the beginning of the hardening and the onset of longitudinal cracks visible along the concrete surface. A higher number of counts and duration are detected, and bigger fluctuations of the $\mathrm{HI}$ are present. The fourth zone is characterized by the propagation of concrete splitting. Longitudinal macro-cracks gradually developed within and on

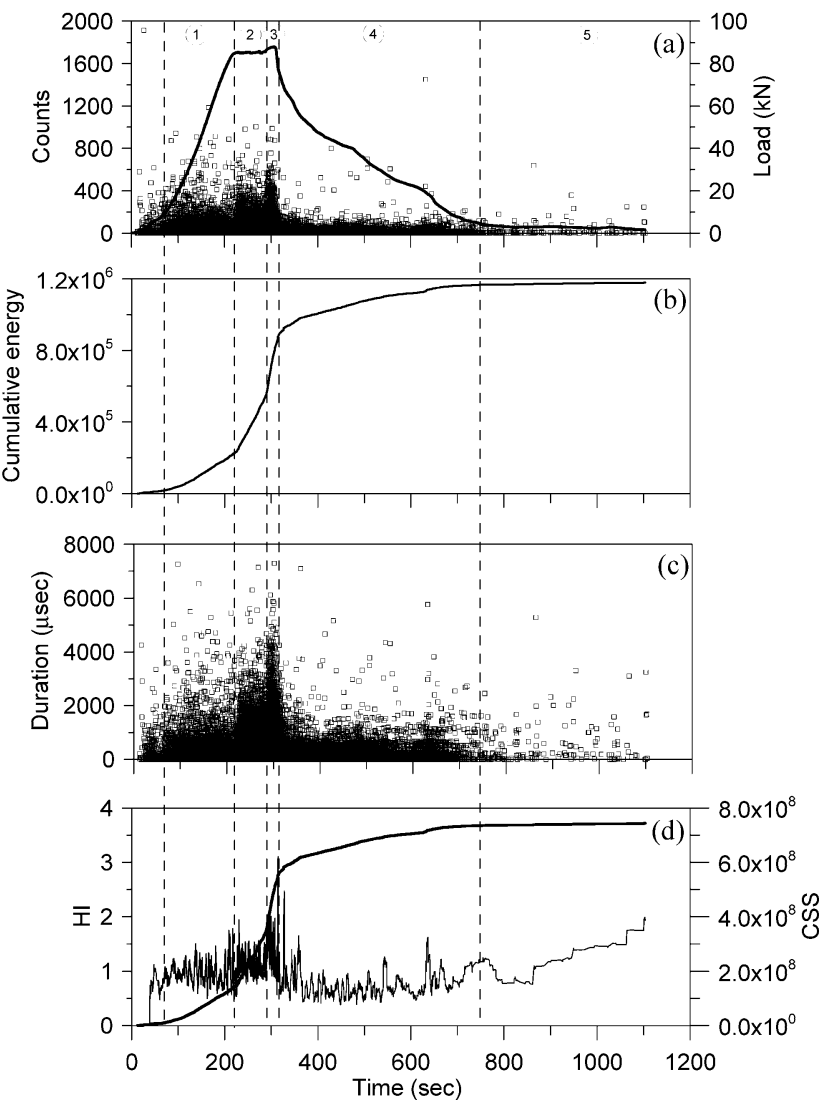

Fig. 10 Test 3-parametric analysis results: (a) counts and applied load, (b) cumulative energy, (c) duration, and (d) historic index and cumulative signal strength as a function of time

the concrete surface. The result is a softening branch associated with the smaller amount of concrete able to sustain the pullout. A lot of AE activity is visible, but with shorter 
Fig. 11 Test 5: moment tensor results. $0 \mathrm{sec}<T<200 \mathrm{sec}$ :

(a) $x-y$ view, (b) $x-z$ view.

$200 \mathrm{sec}<T<450 \mathrm{sec}$ : (c) $x-y$ view, (d) $x-z$ view.

$T>450$ sec: (e) $x-y$ view,

(f) $x-z$ view. $(+)$ sensor's position; $(\diamond)$ shear cracks; ( $\square$ ) tensile cracks; (o) mixed cracks
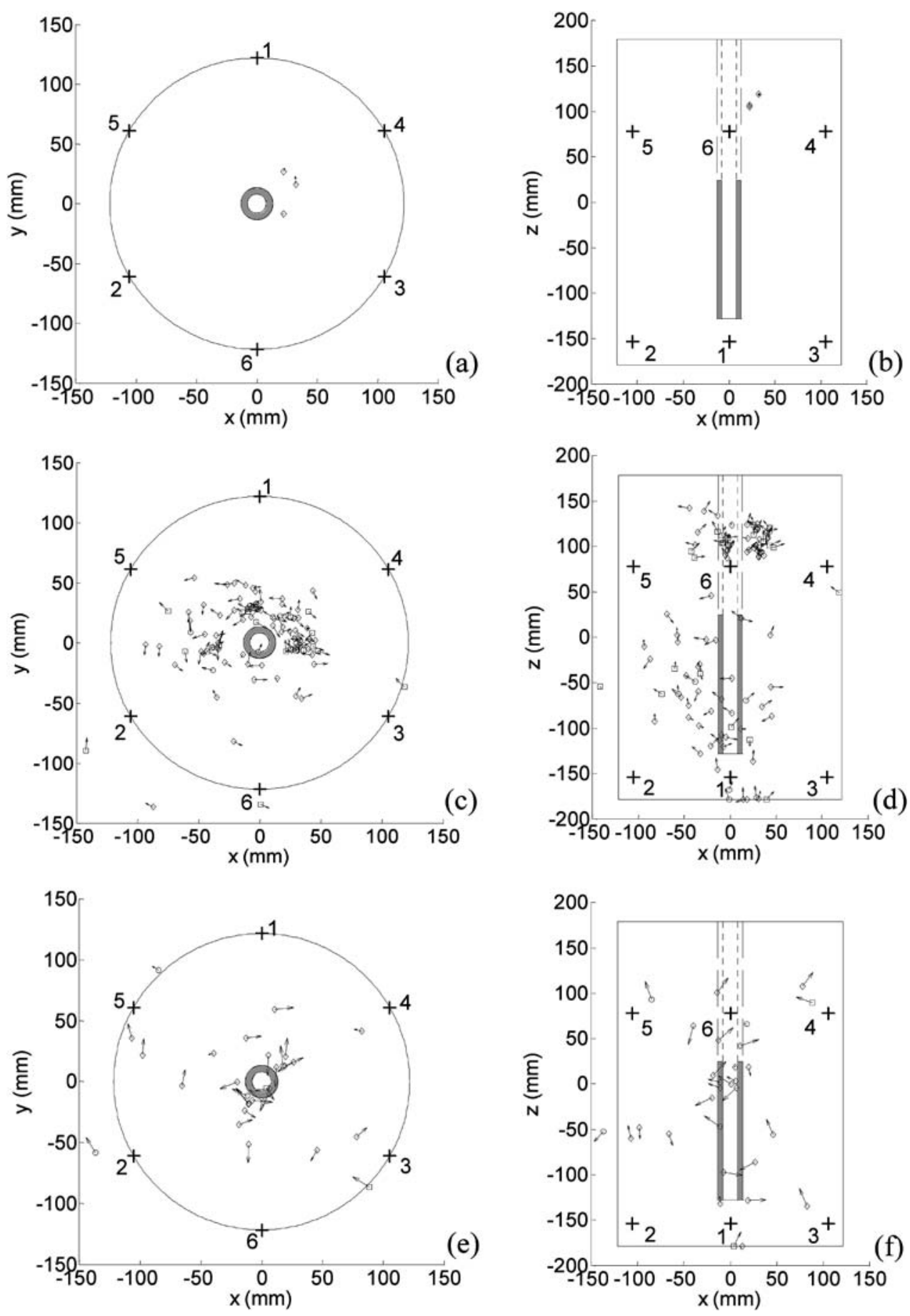

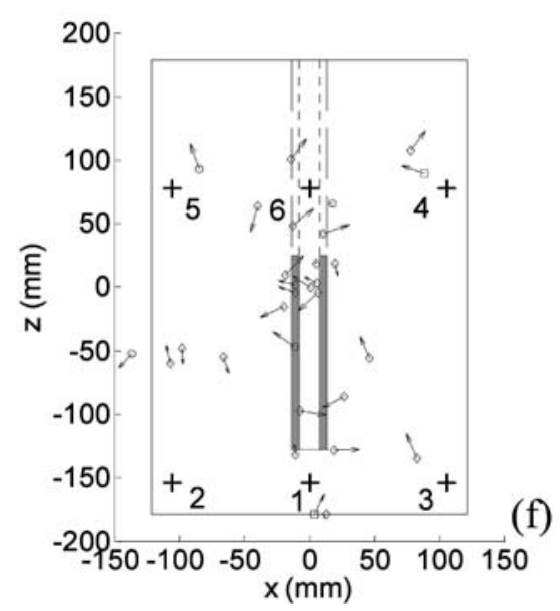

duration and lower numbers of counts. Both the slope of the cumulative energy and the CSS changes gradually until a plateau is reached. The plot of the HI shows short fluctuations.

\section{Experimental Results: Moment Tensor Analysis}

The moment tensor analysis was applied on AE data monitored during Tests 5 and 6 . As shown in Fig. 4, channels 1-3 and 4-6 were placed at $25 \mathrm{~mm}$ from the bottom and $100 \mathrm{~mm}$ from the top of the concrete cylinder, respectively.

The load-displacement curves of Fig. 5 shows that during Test 5 the rebar reached its yielding and suddenly the softening branch initiated. This indicates that debonding occurred at the rebar/resin interface.

Figure 11 shows the results of the moment tensor analysis at three separate time intervals for Test 5. By applying the known relationship between the longitudinal wave velocity 
and the elastic properties of the bulk material [27]:

$v_{p}=\sqrt{\frac{E}{\rho}\left(\frac{1-v}{(1+v)(1-2 v)}\right)}$

where $\rho$ is the material's density, the p-wave velocity of $v_{p}=3133 \mathrm{~m} / \mathrm{s}$ was used.

Shear, tensile, and mixed cracks are identified: a prevalence of shear cracks is present. The arrows indicate the unit crack motion vectors. Overall, the largest amount of cracks is localized around the interface close to sensor 4. Localization errors are mainly related to the approximation of the wave velocity. No significant events were detected during the first 200 seconds. This confirms that the activities detected were mainly associated with the interaction between the grips and the rebar. Diffused activity above the interface was recorded in the $200-450 \mathrm{sec}$ range $(5-13 \mathrm{~mm})$ due to the formation of micro-cracks. Any transient wave generated in the rebar propagated in the steel waveguide and leaks through the interface into the concrete. Such wave was converted into a p-wave and into an s-wave and then detected by sensors $1-6$. The activities clustered near the plane made by sensors 4, 5 and 6 may also be associated with the waves' energy leaking from the rebar.

After $450 \mathrm{sec}$, one main cluster is visible around the bond. At this stage of the experiment, this activity is associable with macro-cracks' formation and the friction between the ribs and the surrounding materials.

Figure 12 shows the position, type, and direction of cracks at three separate time intervals as a result of the moment tensor analysis applied to Test 6 . During this test, the same rebar used for Test 5 is utilized and the bonding length is $102 \mathrm{~mm}$, less than for Test 5 . Because of the Kaiser effect no activities can come from the rebar and the acoustic emissions detected by the six sensors attached in the concrete are attributable to activities at the rebar/resin interface. As expected, cracks are mainly located around the rebar. From the plan view presented in Fig. 12 a and c most of the activities are clustered in the region near sensors 2, 5, and 6 . As for the Test 5, shear cracks are predominant. Comparing the results of the moment tensor analysis occurring at the three time intervals, it is evident that during the softening branch most of the activity is localized at the interface and therefore, it is associated with frictional activities and further onset and propagation of macro-cracks.

\section{Discussion and Conclusions}

In Fig. 13, the load as a function of time and the AE cumulative energy registered by the sensor on the top of the rebar for all the tests are reported. By observing the data associated with systems failed due to pullout shear, it is evident that the cumulative energy associated with Test 2 was higher than Tests 1,5 and 6 . Although not verified by visual inspection, it can be argued that the effective length of the bond was higher than $152 \mathrm{~mm}$. This hypothesis is confirmed by Fig. 13a where the load shows clear indication of yielding and hardening. Such behavior can be attributable to bond length longer than the $152 \mathrm{~mm}$ designed for the test.

As expected, the structural response and the AE behavior of the system in Test 3 differ from the others (or from each other). Such a behavior was the response to gradual concrete splitting.

The sudden failure of concrete during Test 4 determines the sudden drop of the pullout strength visible in Fig. 13a.

Figure 14 reports the intensity chart for all the six tests in the logarithmic scale. The general pattern suggests that low values of the $\mathrm{HI}$ are typical of shear pullout failure happened. The arrows in Fig. 14 denote a movement towards higher values of $\mathrm{AE}$ data associated to the reused rebars. As these rebars must conform to the Kaiser effect, it can be argued that the values associated with Tests 2 and 6 are attributable to main events in the bond. The datum associated with the \#6 rebar has the highest values of $\mathrm{HI}$ and severity. Such a behavior is the result of a simultaneous occurrence of higher rebars strength, bond strength, and concrete failure.

In this paper, the $\mathrm{AE}$ method was used to monitor the pullout test of concrete reinforced with chemically bonded anchors. The test simulated the structural response of adhesively bonded anchor systems used in many structural engineering applications.

The experimental study presented here consists of six pullout tests where single rebar was embedded in hardened concrete cylinders by using polyester resin. Different bond lengths and rebar's sizes were also considered. For one test, an eccentric position of the rebar was considered as well. Tests were carried out in displacement control. Two failure phenomena were observed: shear pullout failure and concrete splitting failure.

Acoustic emission was used with the objective of detecting the onset and propagation of damage and to discriminate the different damage mechanisms. The main causes of possible damage were micro and macro-cracking at the bond interface, grain dislocations in rebar, rebar yielding or hardening, macro-cracking in concrete. The AE events were analyzed using the parametric analysis, the intensity analysis, and the moment tensor analysis. By using the parametric analysis and the intensity analysis, a qualitative discrimination between $\mathrm{AE}$ sources and understanding the structural soundness were obtained. Using the intensity chart areas associated with different dominant events was observed. However, the limited number of specimens studied could not establish a general rule yet and further testing is necessary. In addition, as the intensity analysis is closely related to the 
Fig. 12 Test 6: moment tensor results. $0 \mathrm{sec}<T<600 \mathrm{sec}$ : (a) $x-y$ view, (b) $x-z$ view. $600 \mathrm{sec}<T<1000 \mathrm{sec}$ : (c) $x-y$ view, (d) $x-z$ view. $T>1000$ sec: (e) $x-y$ view, (f) $x-z$ view. $(+)$ sensor's position; $(\diamond)$ shear cracks; ( $\square$ ) tensile cracks; (o) mixed cracks
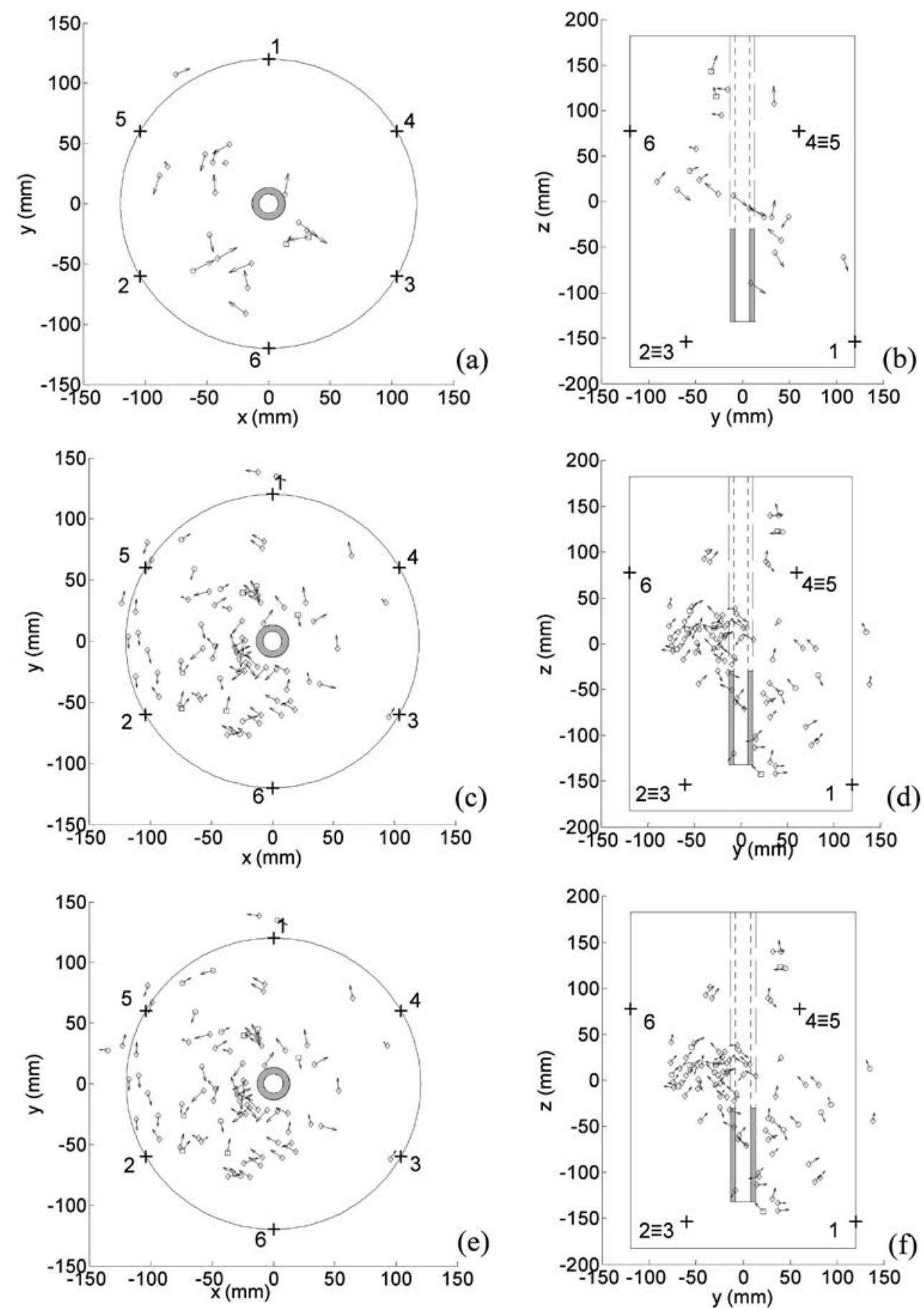

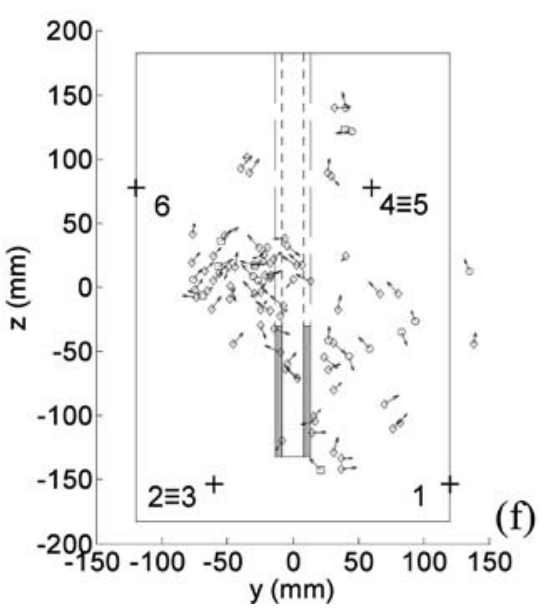

parameter analysis the calculation of the $\mathrm{HI}$ and $\mathrm{Sr}$ may add redundancy to the AE measurements. The simultaneous use of CSS, severity and cumulative energy, as they are related to each other, would imply more knowledge about the source mechanisms than necessary. The moment tensor analysis, instead, provided information both in terms of source localization and damage propagation orientation.

For field testing, it must be pointed out the moment tensor analysis would require the employment of at least six $\mathrm{AE}$ sensors and the configuration adopted in this study could not be always possible.

Acknowledgements The second author conducted this research while visiting University of Pittsburgh under a study Abroad Fellowship of the University of Palermo's School of Engineering. Mr. Degala (former Master's Degree student at the University of Pittsburgh) performed this work under the support of start-up funds available to the first author. The authors are grateful to Dr. Zvi Meiksin at Alertek, LLC for having sparked the interest in this research, and to Dr. Kent A. 

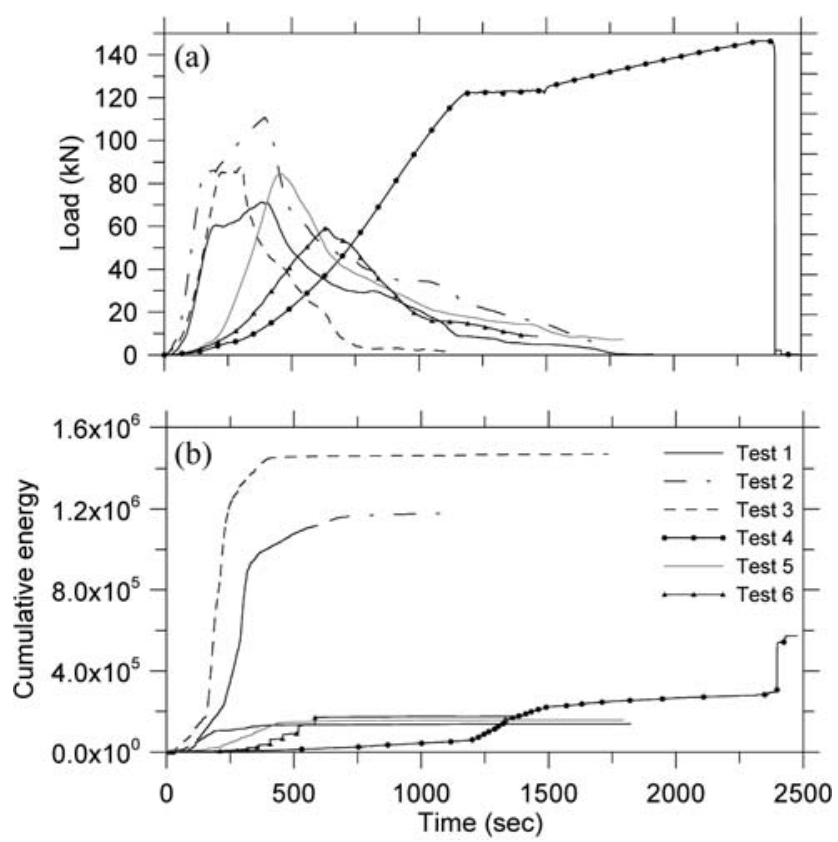

Fig. 13 Load and cumulative energy versus time plots for all the tests

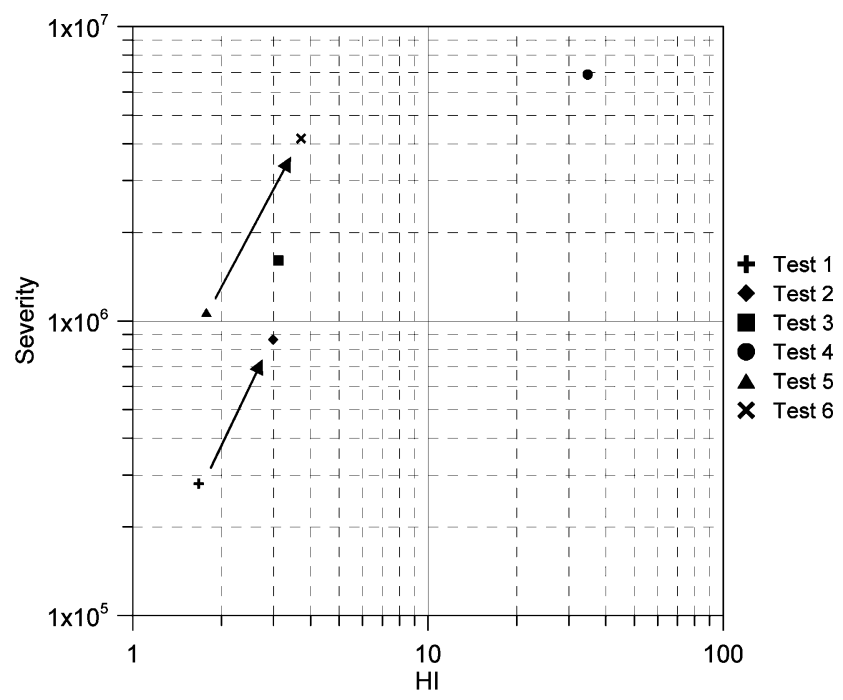

Fig. 14 Intensity chart for all tests discussed in this paper

Harries for helpful discussions during the preparation of the test setup. Finally, Minova Americas it is acknowledged for the donation of the epoxy used in the experiment.

\section{References}

1. National Transportation Safety Board: Ceiling Collapse in the Interstate 90 Connector Tunnel Boston. Massachusetts July 10, 2006, Accident Report NTSB/HAR-07/02 PB2007-916203 (2007)

2. Cheng, C.C., Chiou, H.C.: Evaluating the bond-loss of reinforcing bar in new concrete construction subjected to earthquake using the impact-echo. J. Chin. Inst. Eng. 25, 425-436 (2002)
3. Lin, Y.F., Lin, Y., Tsai, B.Y.: Evaluating bond quality at interface between reinforcing bars and concrete using impact-echo method. Mater. J. 101, 154-161 (2004)

4. Ghandehari, M., Krishnaswamy, S., Shah, S.: Technique for evaluating kinematics between rebar and concrete. J. Eng. Mech. 125, 234-241 (1999)

5. Söylev, T.A., François, R.: Corrosion of reinforcement in relation to presence of defects at the interface between steel and concrete. J. Mater. Civ. Eng. 17, 447-455 (2005)

6. Ito, F., Nakahara, F., Kawano, R., Kang, S.S., Obara, Y.: Visualization of failure in a pull-out test of cable bolts using X-ray CT. Constr. Build. Mater. 15, 263-270 (2001)

7. Pisanova, E., Zhandarov, S., Mäder, E., Ahmad, I., Young, R.J.: Three techniques of interfacial bond strength estimation from direct observation of crack initiation and propagation in polymerfiber systems. Composites, Part A 32, 435-443 (2001)

8. Beard, M.D., Lowe, M.J.S., Cawley, P.: Inspection of rockbolts using guided ultrasonic waves. Rev. Progr. QNDE 20, 1156-1163 (2001)

9. Beard, M.D., Lowe, M.J.S.: Non-destructive testing of rock bolts using guided ultrasonic waves. Int. J. Rock Mech. Mining Sci. 40, 527-536 (2003)

10. Balázs, G.L., Grosse, C.U., Koch, R., Reinhardt, H.W.: Damage accumulation on deformed steel bar to concrete interaction detected by acoustic emission technique. Mag. Concr. Res. 48, 311320 (1996)

11. Fink, F.: Application of the moment tensor inversion in material testing. Otto-Graf-J. 12, 145-156 (2001)

12. Grosse, C.U., Reinhardt, H.W., Fink, F.: Signal-based acoustic emission techniques in civil engineering. J. Mater. Civ. Eng. 15, 274-279 (2003)

13. Köppel, S., Grosse, C.: Advanced acoustic emission techniques for failure analysis in concrete. Institute of Structural Engineering (Zurich, SUI) and Institute of Construction Materials (Stuttgart, GER)

14. Rizzo, P., Lanza di Scalea, F.: Acoustic emission monitoring of carbon-fiber-reinforced-polymer bridge stay cables in large-scale testing. Exp. Mech. 41, 282-290 (2001)

15. Matta, F., Rizzo, P., Karbhari, V., Lanza di Scalea, F.: Acoustic emission damage assessment of steel/CFRP adhesive bonds for rehabilitation. ASCE J. Compos. Constr. 10, 265-274 (2006)

16. Degala, S., Rizzo, P., Ramanathan, K., Harries, K.A.: Acoustic emission monitoring of CFRP reinforced concrete slabs. Constr. Build. Mater. 23(5), 2016-2026 (2009)

17. Gostautas, R.S., Ramirez, G., Peterman, R.J., Meggers, D.: Acoustic emission monitoring and analysis of glass fiberreinforced composites bridge decks. J. Bridge Eng. 10, 713-721 (2005)

18. Landis, E.N., Shah, S.P.: Recovery of microcrack parameters in mortar using quantitative acoustic emission. J. Nondestr. Eval. 12, 219-232 (1993)

19. Ohtsu, M.: Acoustic emission theory for moment tensor analysis. Res. Nondestr. Eval. 6, 169-184 (1995)

20. Suaris, W., Van Meier, J.G.M.: Acoustic emission source characterization in concrete under biaxial loading. Mater. Struct. 28, 444-449 (1995)

21. Chang, S.H., Lee, C.I.: Estimation of cracking and damage mechanisms in rock under triaxial compression by moment tensor analysis of acoustic emission. Int. J. Rock Mech. Mining Sci. 41, 10691086 (2004)

22. Ohno, K., Shimozono, S., Ohtsu, M.: Cracking mechanisms of diagonal-shear failure monitored and identified by AE-SiGMA analysis. Fract. Mech. Concr. Struct. 2, 991-998 (2007) 
23. Malhotra, V.M., Carino, N.J.: Non-destructive Testing of Concrete, 2nd edn. ASTM-CRC, Boca Raton (2004)

24. Cook, R.A.: Behavior of chemically bonded anchors. ASCE J. Struct. Eng. 119, 2744-2762 (1993)

25. Sakla, S.S.S., Ashour, A.F.: Prediction of tensile capacity of single adhesive anchors using neural networks. Comput. Struct. 83, 1792-1803 (2005)
26. Press, W.H., Teukolsky, S.A., Vetterling, W.T., Flannery, B.P.: Numerical Recipes in C, 2nd edn. Cambridge University Press, Cambridge (1992)

27. Shull, P.J.: Non-destructive Evaluation. Theory, Techniques, and Applications. Dekker, New York (2002) 